\title{
Neonatal aspergillus endocarditis: case report and review of literature
}

\author{
Amrita Roy ${ }^{1 *}$, Debadatta Mukhopadhyay ${ }^{2}$, Tamasish Mukherjee ${ }^{3}$, Kaustabh Chaudhuri ${ }^{1}$
}

\author{
${ }^{1}$ Department of Paediatrics, Apollo Gleneagles Hopsitals, Kolkata, West Bengal, India \\ ${ }^{2}$ Department of Paediatric Cardiology, Apollo Gleneagles Hopsitals, Kolkata, West Bengal, India \\ ${ }^{3}$ Department of Cardiothoracic Vascular Surgery, Apollo Gleneagles Hopsitals, Kolkata, West Bengal, India
}

\author{
Received: 17 July 2021 \\ Revised: 16 August 2021 \\ Accepted: 17 August 2021 \\ *Correspondence: \\ Dr. Amrita Roy, \\ E-mail: preences.amri3107@gmail.com
}

Copyright: $\odot$ the author(s), publisher and licensee Medip Academy. This is an open-access article distributed under the terms of the Creative Commons Attribution Non-Commercial License, which permits unrestricted non-commercial use, distribution, and reproduction in any medium, provided the original work is properly cited.

\begin{abstract}
Neonatal fungal endocarditis (FE) remains a rare condition associated with prematurity. It often puts us in diagnostic and therapeutic dilemma as there are no specific guidelines. We described our successful journey with a 26 days old neonate with aspergillus endocarditis responding to multidisciplinary approach with surgical resection and intravenous antifungals.
\end{abstract}

Keywords: Fungal, Endocarditis, Aspergillus, Surgical resection

\section{INTRODUCTION}

Neonatal FE remains a serious complication associated with high morbidity and mortality. ${ }^{1}$ Recommendations from adults/children cannot not be extrapolated to neonates due to different fungal species, cardiac sites, treatment modalities and outcome. ${ }^{2}$ We reported a successful management of aspergillus $\mathrm{FE}$ in a neonate with multimodality therapy.

\section{CASE REPORT}

Twenty six day old, term (40+1 weeks), large for gestational age (birth weight $4.160 \mathrm{kgs})$, male baby was referred due to persistent fever $\left(100-101^{\circ} \mathrm{F}\right)$ for last 2 weeks, not responding to antibiotics. Baby was born to primigravida mother with prolonged labour and shoulder dystocia. The antenatal period was uneventful. Baby had perinatal depression with Apgar scores of 51 and 75 . Umbilical venous catheter was present for 7 days and baby was recovering. However, he developed persistent fever from day 12 with sterile blood culture. Echocardiogram and USG brain were normal.
On examination, baby was non-dysmorphic, febrile, normal saturation, firm hepatomegaly $(5 \mathrm{~cm}$ below right costal margin) and $2 \mathrm{~cm}$ splenomegaly. Blood investigations revealed TLC $14,500 / \mathrm{mm}^{3}$ with raised CRP $(5.9 \mathrm{mg} / \mathrm{dl}$, normal $<0.5)$. Chest X-ray was normal. However, both blood culture and CSF culture were sterile. Urine examination (including fungal hyphae) and TORCH screen were negative. USG abdomen showed coarse hepatic echotexture. Empiric second line antibiotics were continued.

Screening echocardiogram on day 3 of admission showed $15 \times 8 \mathrm{~mm}$ pedunculated heterogenous mass with irregular margins arising from left side of interatrial septum (IAS) with LVEF $70 \%$ (Figure 1). Possibility of vegetation fungal/bacterial/thrombus/left atrial myxoma were considered. Liposomal amphotericin B was started empirically. Repeat blood cultures, eye examination and USG kidneys did not show any evidence of fungal infection. ECG and MRI brain were normal. On further serial echo, the mass extended to the right side of IAS through patent foremen ovale (PFO) (Figure 2).

Considering the high risk of embolization from the large friable mass, cardiotomy and mass resection was done on 
day 14 of admission. Postoperatively, baby needed adrenaline, dobutamine and milrinone infusion. BAL was also negative for fungus. Baby was extubated to room air on day 3 post-op.

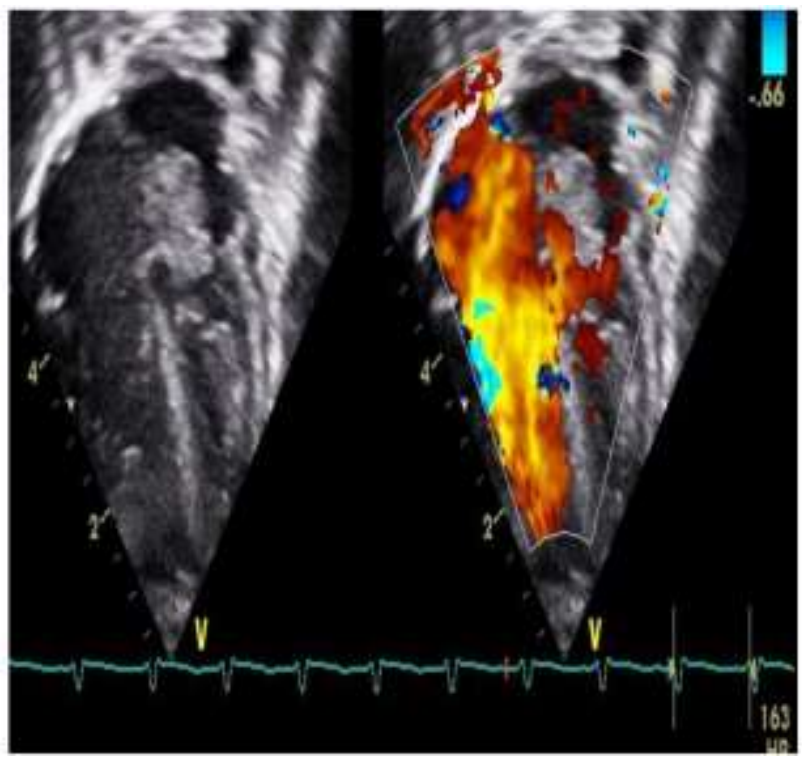

Figure 1: Echocardiograhic picture of left atrial irregular variegated mass at diagnosis.

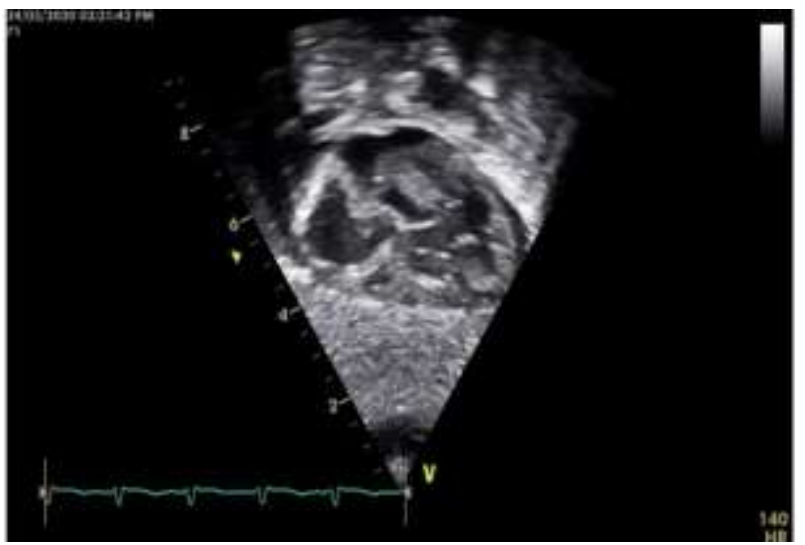

Figure 2: Echocardiographic picture of extension of left atrial mass to right atrium.

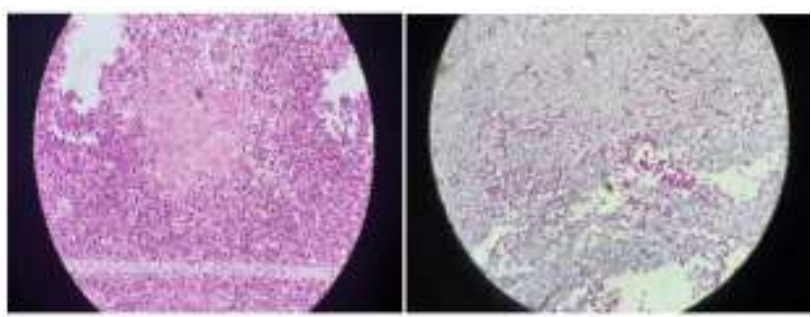

Figure 3: Haematoxylin and eosin stain showing fibrin necrotic debris with mixed inflammatory cells and multinucleated giant cells; PAS stain showing septate hyphae and acute angle branching form of Aspergillus spp
Histopathological examination of the mass with haematoxylin and eosin revealed acute angled branching septate hyphae suggestive of aspergillus with fibrin necrotic debris, inflammatory cells and giant cells (Figure 3 ). Culture of the mass was sterile. IV voriconazole was added to liposomal amphotericin $\mathrm{B}$ and both were continued for 6 weeks followed by oral prophylaxis along with aspirin.

In view of deep seated infection with atypical organism, screening for primary immunodeficiency was done which was negative. Baby was discharged at 3 months with intact neurodevelopmental outcome. Serial echocardiogram has been normal till date (1.5 year follow up).

\section{DISCUSSION}

Incidence of infective endocarditis (IE) in children was between 0.8 and 3.3 per 1000 paediatric admissions. ${ }^{3} \mathrm{FE}$ ranged between $0-12 \%$ of pediatric IE. In a review by Ganesan et al $48 \%$ of paediatric FE were infants of which $61 \%$ were preterm neonates with median GA of 27 weeks and median birth weight of 860 grams. $^{4}$

The pathogenic mechanism for FE was presence of thrombus on traumatized endocardium, on which the fungi circulating in the bloodstream colonize. ${ }^{5}$ Risk factors included prematurity $(92 \%)$, prior antibiotics $(81 \%)$, central venous catheters $(71 \%)$, prior or concurrent bacteremia (22\%), congenital heart disease $(13 \%)$, prior surgery $(13 \%)$. Our patient had prior broadspectrum antibiotic use, UVC insertion and PFO. ${ }^{5}$

The clinical presentation of FE in neonates was mostly nonspecific (similar to sepsis). In a review by Dorothea et al right atrium was involved in $63 \%$, right tricuspid valve (24\%), pulmonic valves (7\%) and multifocal $(45 \%){ }^{6}$ Multiorgan involvement was reported in $21 \%$ with kidneys (12\%), CNS (7\%), liver (4\%), skin (3\%) and spleen with lung $(1 \%){ }^{6}$ In our patient, left atrium was first involved presenting at atypical site which increased the diagnostic dilemma.

As per the review by Millar et al Candida spp. were the most frequent (63\%) with Candida albicans (41\%). Aspergillus spp. were seen in $26 \%$ with A. fumigatus $(8 \%){ }^{1}$

The diagnosis of FE was difficult. Reliable growth in blood culture was difficult to obtain. Blood cultures of Aspergillus were negative in over $50 \%$ of patients with aspergillus endocarditis. Serologies (mannan and galactomanna) were not reliable. Cultures and PCR from the infected site remain the gold standard but it was invasive. ${ }^{4}$

The American heart association and infectious diseases society of America (IDSA) advocated surgery in conjunction with antifungal treatment. For candida FE, amphotericin B (AMB) \pm 5 flucytosine was first line 
followed by fluconazole as 2 nd line. ${ }^{7}$ According to the European society of clinical microbiology and infectious diseases (ESCMID), patients with native valve candida endocarditis should undergo surgical treatment within 1 week combined with liposomal amphotericin B or caspofungin for 6-8 weeks \pm additional flucytosine. ${ }^{8}$

Treatment of aspergillus FE needed early recognition followed by rapid surgical resection with antifungal therapy because of the high risk of embolic complications and cardiac decompensation. IDSA recommended voriconazole as the preferred agent. Alternatively, liposomal amphotericin B could be administered alone or in combination with voriconazole for at least 6 weeks after surgery. ${ }^{9}$ Itraconazole and caspofungin were reserved for refractory aspergillosis with lifelong triazole prophylaxis following prosthetic valve replacement. Our patient responded well to amphotericin $\mathrm{B}$ and voriconazole combination for 6 weeks.

Timing of surgical intervention depended on risk of disseminated infected emboli, increased vegetation mobility and enlargement while on treatment, hemodynamic instability, valve dehiscence and perivalvular abscess. ${ }^{10}$

The overall case fatality rate was $42.2 \%$ and $64 \%$ for multiorgan involvement. Infection with yeast was associated with significantly better outcomes (42\%) when compared with moulds $(65 \%, \mathrm{p} \leq 0.05) .{ }^{4}$ The recurrence rate was very high, with fatal prognosis. An early diagnosis and appropriate treatment were essential to maximize survival rates.

\section{CONCLUSION}

Paediatrician must suspect FE in high-risk groups and aim to detect of the causative organism. Combination of antifungal therapy and surgical debridement is the preferred treatment. Though fatality in this condition is reported to be high, early diagnosis and multimodality treatment can lead to a successful outcome.

\section{ACKNOWLEDGEMENTS}

Authors would like to thank parents of the baby, nursing staff at Apollo Gleneagles hospitals, Kolkata.

Funding: No funding sources Conflict of interest: None declared

Ethical approval: Not required

\section{REFERENCES}

1. Millar BC, Jugo J, Moore JE. Fungal endocarditis in neonates and children. Pediatr Cardiol. 2005;26(5):517-36.

2. Yuan SM. Fungal Endocarditis. Braz J Cardiovasc Surg. 2016;31(3):252-5.

3. Humpl T, McCrindle BW, Smallhorn JF. The relative roles of trans-thoracic compared with transesophageal echocardiography in children with suspected infective endocarditis. J Am Coll Cardiol. 2003;41(11):2068-71.

4. Ganesan V, Ponnusamy SS, Sundaramurthy R. Fungal endocarditis in paediatrics: a review of 192 cases (1971-2016). Cardiol Young. 2017;27(8):1481-7.

5. Shane AL, Stoll BJ. Recent developments and current issues in the epidemiology, diagnosis, and management of bacterial and fungal neonatal sepsis. Am J Perinatol. 2013;30(2):131-41.

6. Pana ZD, Dotis J, Iosifidis E, Roilides E. Fungal endocarditis in neonates: a review of 71 cases (1971-2003). Pediat Infect Dis J. 2015;34(8):803-8.

7. Pappas PG, Kauffman CA, Andes D, Benjamin DK, Calandra TF, Edwards JE, et al. Infectious diseases society of America. Clinical practice guidelines for the management of candidiasis: 2009 update by the infectious diseases society of America. Clin Infect Dis. 2009;48(5):503-35.

8. Cornely OA, Bassetti M, Calandra T, Garbino J, Kullberg BJ, Lortholary O, et al. ESCMID fungal infection study group. ESCMID guideline for the diagnosis and management of candida diseases 2012: non-neutropenic adult patients. Clin Microbiol Infect. 2012;18(7):19-37.

9. Walsh TJ, Anaissie EJ, Denning DW, Herbrecht R, Kontoyiannis DP, Marr KA, et al. Treatment of aspergillosis: clinical practice guidelines of the infectious diseases society of America. Clin Infect Dis. 2008;46(3):327-60.

10. Nomura F, Penny DJ, Menahem S, Pawade A, Karl TR. Surgical intervention for infective endocarditis in infancy and childhood. Ann Thorac Surg. 1995;60(1):90-5.

Cite this article as: Roy A, Mukhopadhyay D, Mukherjee T, Chaudhuri K. Neonatal aspergillus endocarditis: case report and review of literature. Int J Contemp Pediatr 2021;8:1612-4. 